Journal of Advanced Computer Science \& Technology, $8(1)(2019) 1-10$
Journal of Advanced Computer Science \& Technology
SPC
Website: $w w w . s c i e n c e p u b c o . c o m / i n d e x . p h p / J A C S T$
Research paper

\title{
Investigation of interference noises frequencies of human heart
}

\author{
Kadim Karim Mohsen ${ }^{1 *}$, Abdul Gaffer. S .M ${ }^{2}$, Satar Habib Mnaathr ${ }^{2}$ \\ ${ }^{1}$ Department of Mechanical Engineering, Engineering College, University of Thi-Qar, Nasiriya, Iraq \\ ${ }^{2}$ Department of Electrical and Electronic Engineering, Engineering College, University of Thi-Qar, Nasiriya, Iraq \\ *Corresponding author E-mail: Usm111usm@gmail.com
}

\begin{abstract}
Electrical incitation Starts in the center of bunches called center sinoatrial masterminded in the left chamber and a brief span later moves to the atria and beginning there to the accompanying center point is known as a center point atrioventricular organized in contact chamber and left the region in the left ventricle and after that to the bundle, and a while later to the ventricles and are plotted this begin which happens in the heart by a contraption (ECG) and are gotten development by Alake roach where when the pickup isn't just get the sign made by the heart muscles just besides signals working out as intended because of different muscles of the body Calcar and arms, and also the signs passed on by the boundary, and after that pass the sign on where the channel is relied upon to expel such vexatious frequencies. ECG accounts are routinely debased by high repeat unsettling influences. for instance, electrical mechanical assemblies and these signs negatively affect the chart which drew devices ECG, EEG and EMG electrical connection impedance electromyography and in resembling mode not having the ability to examine it and center the case of the patient must be the departure of these signs, known frequencies by high We have used the underlying two schedules are the channel and we used adaptable noise canceller (ANC) and the second is by MATLAB program which has been stacked banner and adherent it to modernized banner by Fourier Transform thus to make it less requesting to scrutinize and after that pass the sign on where the channel is expected to remove such bothersome frequencies. The convenience of this approach is demanded by utilizing imitated ECG and Adaptive channels have been generally utilized as a bit of the withdrawal of confusion in biomedical standards.
\end{abstract}

Keywords: Frequency Noise; ECG Signal; Biomedical Signals; Physiology of the Heart.

\section{Introduction}

A large portion of the strategies depend upon outer reference signals which are connected with the noise in the fundamental data. For the annals of biomedical signs with fuss inside and can't be known from the previously, the utilization of outer reference is dicey. Moreover, for the condition where the outer reference signals unequivocally connected with the bid can't be conceivable a structure without the need of outside reference is required. Adaptable noise canceller (ANC) for high-repeat disturbance in ECG without the need of reference signs is tended to in this paper. Essential lead behind this is to misuse the property of periodicity existed in the ECG. Besides, we utilized the new strategy to settling the high repeat in ECG context by operation tangle lab properties through system new station with particular characteristic to joined two sorts of sign and faultless pennant and change hail by then utilize the odder to clear the noise.

Electrical connection obstacle electromyography (EMG) disturbance, and instrumentation aggravation coordinates by the use of adaptable in scratching off the change requires an outside reference to survey the tumult and, thus subtracting it from the uproarious ECG. Notwithstanding, this is a boundless part of the time lacking because of the way that the reference signal can't be all around related with the unsettling influence part in the basic data. A versatile structure used to manage the clatter without the need of outside reference is Addressed in this paper. Essential thought behind this is an immediate aftereffect of the way that ECG signs can be overseen as discontinuous signals in a few frequencies. This sorts it conceivable for a versatile channel to study ideal ECG movement from the uproarious one. Electrical mechanical gatherings and these signs adversely influence the outline which drew utensil (ECG) and in like way not being able to analyze it and focus the instance of the patient must be the takeoff of these signs, known frequencies of high fundamental two modes are the channel utilized (adaptable channel) and the second is by MATLAB program which has been loaded standard and follower it to modernized pennant by Forel Transform along these lines to make it less asking for to investigate and after that pass the sign on where the channel is relied upon to evacuate such troublesome frequencies.

The standard ECG is by exclusively constrained to $0.05-150 \mathrm{~Hz}$, in any case higher frequencies are in like way present in the ECG signal. With high-confirmation advancement, it is conceivable to record and break down these higher frequencies. The most astonishing amplitudes of the high rehash pieces are found inside the QRS complex [1]. In this examination, it was likewise displayed that the developments found in HF-QRS in patients with myocardial ischemia may be an aftereffect of the coordinating of the conduction speed in the district of ischemia [2]. This section has been endeavored by Watanabe et al [3] by ingraining sodium channel blockers into the left front diving supply course in pooches. In their examination, 60 unipolar 2 ECGs were recorded from the whole ventricular surface and were sign found the middle estimation of and separated in the $30-250 \mathrm{~Hz}$ rehash go. comes to fruition demonstrated that the decline noted in 
the HF-QRS related straightly with the territory conduction delay. Benn Hagen et al [4] demonstrated that root mean square (RMS) voltage estimations of the depolarization sign relate insufficiently with the sign plentifulness yet exceedingly with the first and second assistants, i.e. the speed and the extending speed of the sign. It has in like way been recommended that the autonomic unmistakable system impacts HF-QRS. sitting up reasons fundamental changes in HF-QRS in a few leads separated from the prostrate position [5]. Evaluation of HF-QRS). Athletic people, particularly world class contenders, who have vagally-intervened changes on the ordinary ECG (i.e. early repolarization, bradycardia) have broadened RAZ change [6].

\section{Anatomy and physiology of the heart}

The heart is masterminded in the waist between the lungs behind the sternum or progressively the stomach. It is encompassed by the pericardium. Its size talks reality that of a grip hand, and its weight talks reality $250-300 \mathrm{~g}$. Its middle is organized around $1.5 \mathrm{~cm}$ to the opposite side of the mid sagittal plane. Masterminded over the heart are the titanic vessels: the inescapable and beneath normal vena cava, the aspiratory supply course and vein, and moreover the aorta. The aortic curve lies behind the heart. The throat and the spine lie help behind the heart [12]. Fig.1. show the life structures of the heart and related vessels.

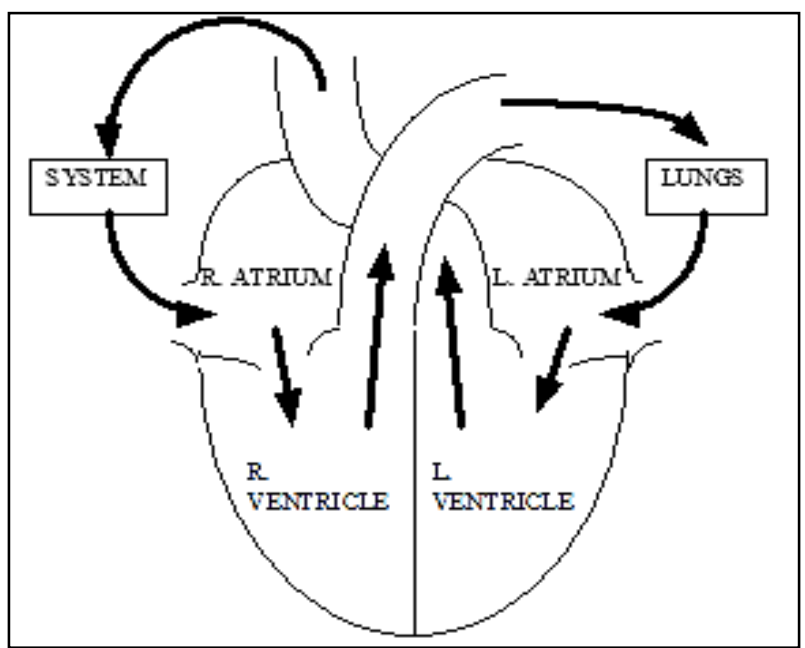

Fig. 1: The Anatomy of the Heart and Associated Vessels.

\section{Electric action of the heart}

\subsection{Cardiac muscle cell}

The sufficiency of the movement potential is also tantamount, talking reality $100 \mathrm{mV}$ for both nerve and muscle. The term of the heart muscle inspiration is, then again, two solicitations of degree longer than that in either nerve cell or skeletal muscle. A level stage takes after heart depolarization, and starting there repolarization happens. In the nerve cell, repolarization is a consequence of the overflowing of potassium particles. The timeframe of the movement inspiration talks reality $300 \mathrm{~ms}[13,14]$ as in Fig.2. Associated with the electric start of cardiovascular muscle cell is its mechanical withdrawal, which happens to some degree later. A basic refinement between heart muscle tissue and skeletal muscle is that in cardiovascular muscle, start can multiply be beginning with one cell then onto the following in any course. In this manner, the incitation wave fronts are of rather complex shape.

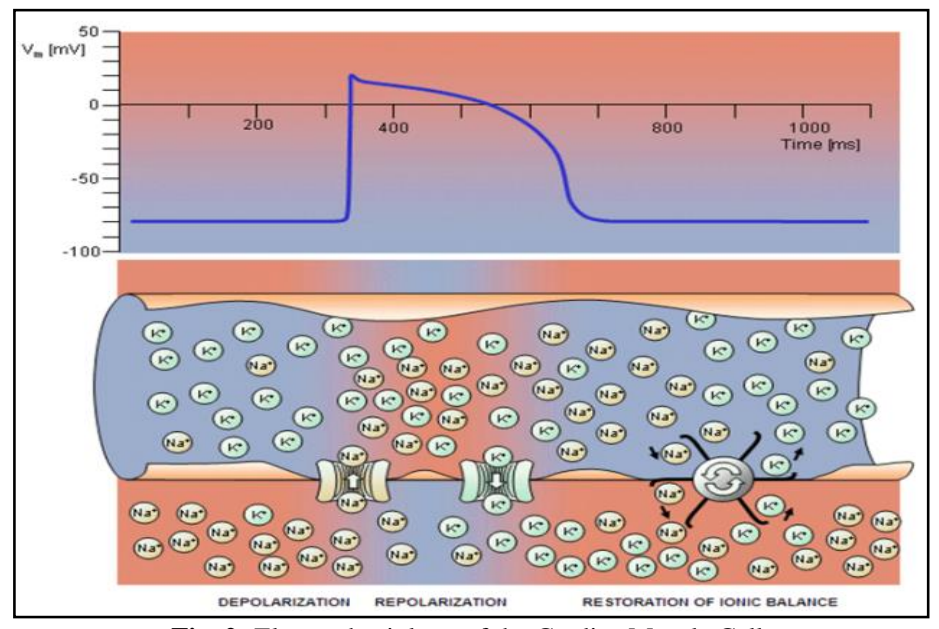

Fig. 2: Electrophysiology of the Cardiac Muscle Cell. 


\section{The conduction system of the heart}

Organized in the right chamber at the unrivaled vena cava is the sinus focus point (Sinoatrial or SA focus point) which incorporates specific muscle cells. The sinoatrial focus point in people is fit as a fiddle of a bow and talks reality $15 \mathrm{~mm}$ long and $5 \mathrm{~mm}$ wide watch Fig. 3 . In a typical heart, the AV focus point gives the essential driving way from the atria to the ventricles. Along these lines, under regular conditions, the last can be engaged just by heartbeats that duplicate through it. Spread from the AV focus point to the ventricles is given by a specific conduction framework. Proximally, this framework is made out of a typical pack, called the mound [15]. All the more distally, it withdraws into two pack branches duplicating along each side of the septum, constituting the advantage and left package branches. (The left package thusly sections into a front and back branch). Widely more distally the social occasions ramify into Purkinje filaments [named after Jan Evangelista Purkinje [16]. Causing along the conduction structure happens at an appropriately brisk once it is inside the ventricular district, at any rate before this (through the AV focus point) the speed is inconceivably immediate. From the internal side of the ventricular divider, the different begin objectives cause the arrangement of a wave front which spreads through the ventricular mass toward the external divider. This system occurs as a result of cell-to-cell enactment. After each ventricular muscle region has depolarized, repolarization happens. Repolarization isn't a spreading wonder, and in light of the way that the time allotment of the activity drive is significantly shorter at the epicedium (the outside side of the cardiovascular muscle) than at the endocardium (the inside side of the heart muscle), the complete of action seems similarly so to speak increasing from epicedium toward the endocardium.

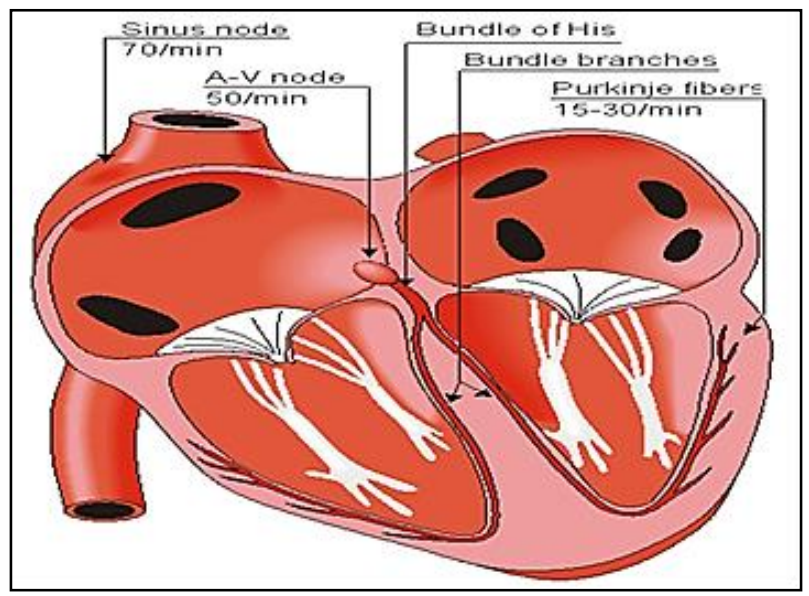

Fig. 3: The Conduction System of the Heart.

\section{ECG waveform fig.4}

$\mathrm{P}$ wave: is the consequence of activity potential reason depolarization of the atrial myocardium. QRS complex: is made out of three individual waves, is result from ventricular depolarization and signs the beginning of ventricular compression.

$\mathrm{T}$ wave: speak to repolarization of the ventricles and goes before ventricular unwinding.

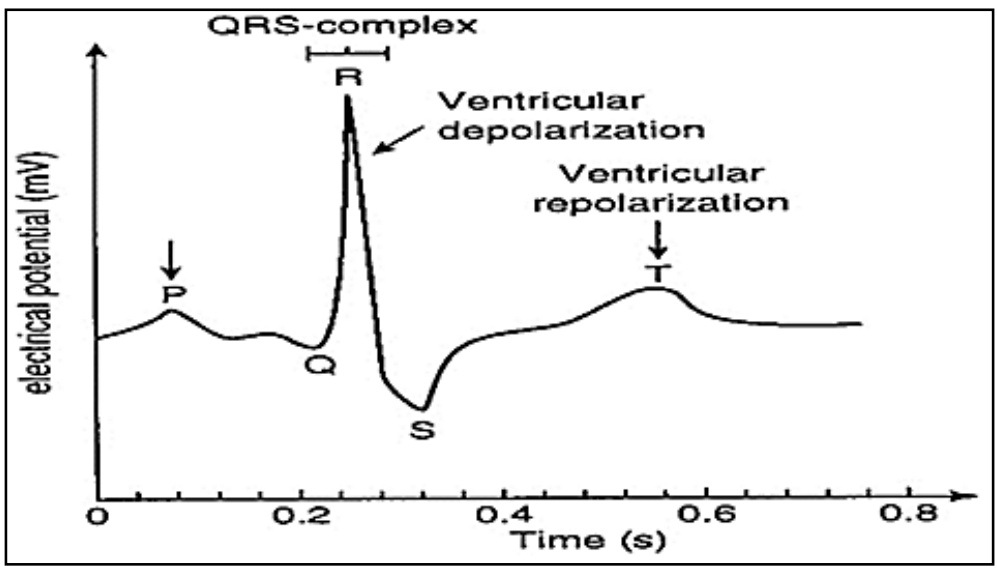

Fig. 4: ECG Waveform.

\section{System analysis}

\subsection{ECG system}

Electrocardiography ECG is a transthoracic (over the thorax or waist) appreciation of the electrical movement of the heart over a traverse of time, as saw by cathodes related with the surface of the skin and recorded by a contraption outer to the body. Heart beat commonness and the level of the chamber can be focus by utilizing ECG. The electrocardiography in like way used to expressive or found any naughtiness of the heart or impact whatever other part, for example, medication or contraptions like pacemaker may besides be performed on creatures, when in doubt for complete of heart assortments from the standard or examination. The (ECG) devise structure is shown on Fig.5. which include on five stages, each stage is as following:1-The principle arrange is a transducer- $\mathrm{AgCl}$ anode, which change over 
ECG into electrical voltage.2-The second stage is an instrumentation intensifier to create information hail 3-Channel to empty vexatious standard 4-Finale speaker to grow the disengaged sign 5-Oscillosco.

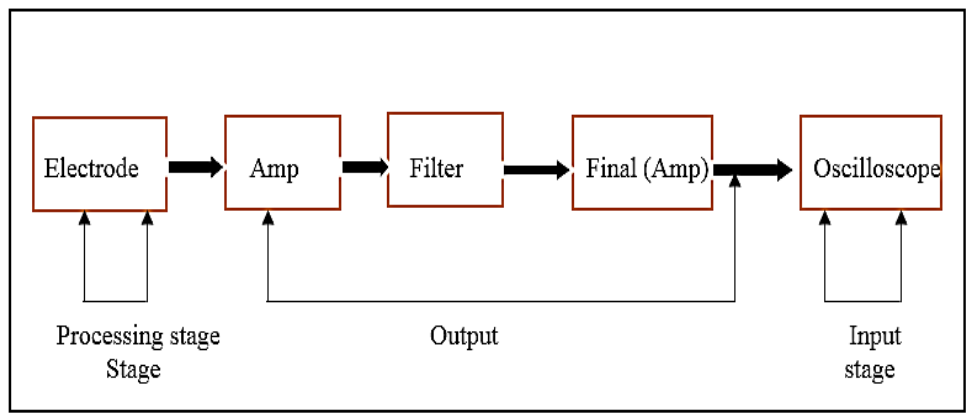

Fig. 5: ECG Block Diagram.

The revenue of an ECG recorder is a framework (or now and again two or three charts, tending to each one of the leads) with time tended to on the x-turn and voltage tended to on the y-focus point. A submitted ECG machine would when in doubt print onto diagram paper which has a foundation instance of $1 \mathrm{~mm}$ squares (routinely in red or green), with striking divisions each $5 \mathrm{~mm}$ in both vertical and even headings. Evaluating at a varying area of the heart will recover specific potential. Furthermore, with the target that it will make different ECG waveforms. The ECG made by each cardiovascular cycle thick on Table.1.

A-Lead: In electrocardiography there are different terminals called lead or in the long run it can be causes much befuddle in light of the way that can be utilized to suggest 2 things. To the rejection of everything else may be known as the electrical association joining the anodes to the ECG recorder or it might be the "left arm lead" as the cathode (and its association) that ought to be related at or close to the other side arm.

Table 1: Duration and Characteristics of Each Major Event in the Cardiac Cycle

\begin{tabular}{lll}
\hline Event & Characteristics & Duration at 0.8 sec/cycle \\
\hline Atrial diastole Ventricular diastole & AV valves opened. Semilunar valves close. Ventricular filling. & 0.4 seconds \\
Atrial systole, Ventricular diastole & AV valves open. Semilunar valves shut. Ventricular filling. & 0.1 seconds \\
Atrial diastole & AV valves shut. Semilunar valves open Blood drew into aorta and aspiratory artery. & 0.3 seconds \\
Ventricular systole & & \\
\hline
\end{tabular}

B-Placement anode: ECG erection require Ten cathodes to join with 12-lead of ECG. We can portray every terminal include a planning gel, which set amidst a self-bond cushion onto which joins cut. In addition, the gel comparatively makes a firm. They are named and put on the patient's physique as follows in the Table.2.

Table 1: Lead Connection for Traditional ECG

\begin{tabular}{ll}
\hline Electrode label (USA) & Electrode placement Electrode placement \\
\hline RA & On the right arm, avoiding thick muscle. \\
LA & In the same location where RA was placed, but on the left arm. \\
RL & On the right leg, lateral calf muscle. \\
LL & In the same location where RL was placed, but on the left leg. \\
V1 & Fourth intercostal space (between ribs 4 and 5) just to the right of the sternum (breastbone). \\
V2 & In the fourth intercostal space (between ribs 4 and 5) just to the left of the sternum. \\
V3 & Between leads V2 and V4. \\
V4 & In the fifth intercostal space (between ribs 5 and 6) in the mid-clavicular line \\
V5 & Horizontally even with V4, in the left anterior axillary line. \\
V6 & Horizontally even with V4 and V5 in the maxillary line. \\
\hline
\end{tabular}

\section{Silver chloride electrode}

Allusion anode which has unfathomable quality and terminal potential, this high dauntlessness begins from the fixation in both side of each section in the redox response for instance silver chloride cathode can be consider as one of these terminal which is normal exploited as a bit of electrochemical estimations. As far as possible as a redox terminal and the response is between the silver metal ( $\mathrm{Ag}$ ) and its salt - silver chloride ( $\mathrm{AgCl}$, in like way called silver (I) chloride, or an overall reaction can be written:

$\mathrm{Ag}^{+}+\mathrm{e}^{-} \leftrightarrow \mathrm{Ag}(\mathrm{s})$

$\operatorname{AgCl}(\mathrm{s}) \leftrightarrow \mathrm{Ag}^{+}+\mathrm{Cl}^{-}$

$\mathrm{AgCl}(\mathrm{s})+\mathrm{e}^{-} \leftrightarrow \mathrm{Ag}(\mathrm{s})+\mathrm{Cl}^{-}$

\section{Applications of silver/sliver chloride}

Locus anodes made by a pliable tube as silver wire that is secured with a dainty layer of silver chloride, the silver wire will be liquid in silver chloride, other way we can set it up misleadingly by utilizing electroplating procedure when the wire concentrated hydrochloric dangerous as Fig (6) underneath demonstrated the critical parts of $\mathrm{Ag} / \mathrm{AgCl}$

Cathode: 


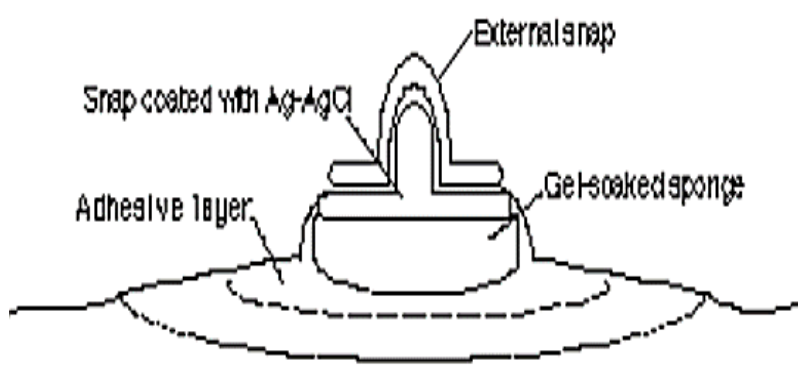

Fig. 6: A Disposable Surface Electrode.

\section{Analysis by using preamplifier}

\subsection{Instrument intensification and DC section}

Regardless of the way that the strong instrumentation speaker with particularly symmetric could decrease the dynamic devices used to shape a three activity amp structure to get high data impedance and incredible fundamental mode rejection. There is still some condition in which it is as versatile as the immense three task amp approach since it is settled. In certifiable utilization of ECG getting, the sensors are consistently achieved the skin through an electrically-conductive gel [17]. The anode skin interfaces are believed to be possible wellspring of plenitude noise; both the gelled surface of the terminal (metal electrolyte) and the interface from gel to skin (electrolyte/electrolyte) may act like a voltaic cell (indicated charmed). In spite of the way that the made voltages at the two cathodes have appropriate furthest point and should to some degree scratch off each other, poor modify may realize waiting commotion [18]. This could make the enhancer drenched without DC prevent in the front-end circuit. One plan is to introduce a high-pass divert in a front of instrumentation enhancer, yet the cost is the decreasing of the data impedance.The forefront grasps a twofold task amp OPA2277 with the features as high open hover get. High impedance and high CMRR to build up the main arrange as parallel related noninverting intensifier as showed up in Fig.7.

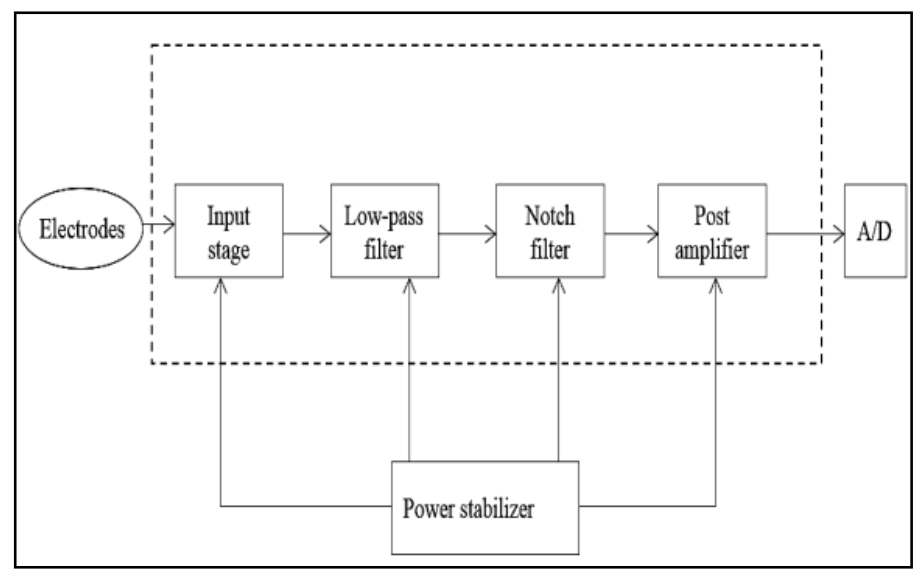

Fig. 7: Analysis by Using Preamplifier.

Since the two task amp in a comparable chip have indistinct characteristics. Some corruption of CMRR on account of fragments mishandle could be avoided. The get of the primary enhancer organize should not be too high, and in this layout it set as $(\mathrm{G}=1+2 \mathrm{R} 1 / \mathrm{R} 2=41$. Capacitors C15 and C16 and resistor R1 and R2 shape the DC square circuit with a cutoff-repeat of $\mathrm{fL}=1 /(2 \pi \mathrm{R} 16 \mathrm{C} 15)=0.07 \mathrm{~Hz}[19]$, which can't be executed in the instrumentation intensifier. The differential improvement organize is fulfilled by the stuffed instrumentation intensifier (AD620, high open hover get, high impedance and high CMRR, etc). The get is changed definitely with a lone external resistor as $\mathrm{G}=49.4 \mathrm{k} \omega / \mathrm{RG}+1$; where $\mathrm{RG}$ is $\mathrm{R} 23$. If $\mathrm{R} 23$ is adjusted in the region of $1 \mathrm{k} \omega$ and $10 \mathrm{k} \omega$, the get of the second enhancer compose $\mathrm{G} 2$ (i.e. $\mathrm{G}$ in the latest condition) will vary from 6 to 50. By then the get of the data stage could be managed from 246 to 2050 .

\section{Common-mode driving circuit}

The normal mode driving circuit, as showed up in Fig.8, is produced by two practically identical resistors R7 and R8, and furthermore the voltage aficionado U2A. Meaning the yield of task amp U1A and U1B as V01 and V02 independently, and Vc the yield of activity amp U2A, by then $\mathrm{Vc}=1 / 2(\mathrm{~V} 01+\mathrm{V} 02)$ [16]. As differential-mode signal input just (i.e., V01=-V02), the midpoint voltage $\mathrm{Vc}=0$, by then the yield of U2A is zero; as differential-mode signal and general mode signal contribution in the meantime, the yield of U2A simply contain the fundamental mode part of the data signals. It suggests that the normal mode signal could be input instrumentation enhancer direct rather than being implied differential-mode agitating impact for screw up of the fragment in the RC coupling circuit [20]. 


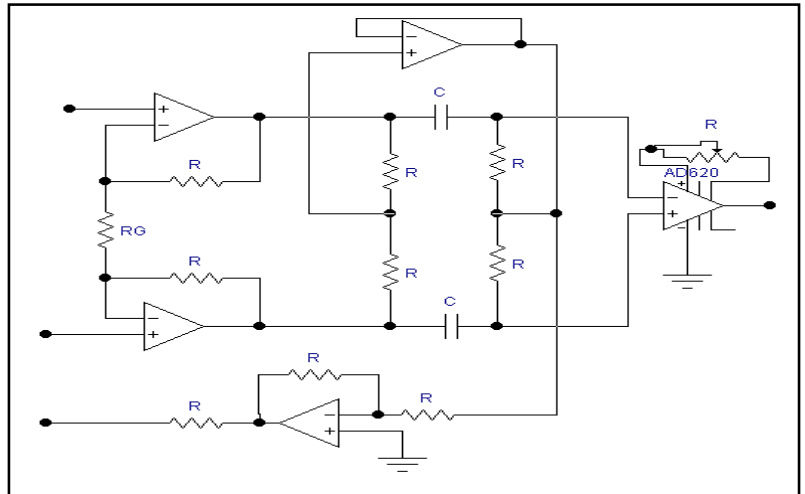

Fig. 8: Schematic of Pre-Amplifier.

\section{1. $\quad 50 H z$ step channel}

By large grasped to settle the electrical link impedance issue including proper building up and electrical securing in straightforward accounts, anyway the preprocessing is major since control line deterrent which overwhelms the pined for signs is as a general rule all around. This changes the customary Twin-T step channel to be Q-think about tunable as showed Fig.9.

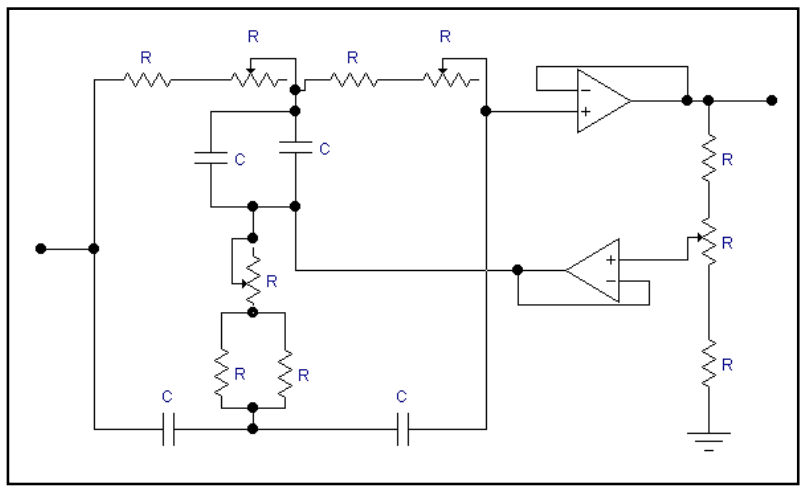

Fig. 9: Circuit of 50Hz Twin-T Notch Filter.

\section{Analysis of HF-QRS}

\subsection{ECG acquisition}

1) Utmost current time ECG frameworks have a sufficiency confirmation of no under $1 \mu \mathrm{V}$, which is satisfactory for examination of HF-QRS change diminishment. The amplitudes of HF-QRS are low $(\mu \mathrm{V})$ showed up distinctively in connection to the amplitudes found in standard ECG $(\mathrm{mV})$. With a specific genuine target to take a gander at HF-QRS, a low racket level is in this manner required. Subsequently the enlistment all finished necessities to continue for several minutes recalling the genuine goal to secure an enough extensive number of QRS structures for averaging. In the event that the ECG morphology is liable to part changes, for instance amidst PTCA, an exponentially (recursively) upgraded beat run of the mill can be utilized rather, since standard piece sharp averaging isn't impeccable in such cases [21,22].

2) Extraction of HF-QRS, this channel genus has a non-straight stage reaction, which may ravage transient association of the particular sign parts. Facilitate arrange sifting can be grown, regardless, by first shedding the sign advancing and a brief span later in reverse Unmistakable examinations have utilized distinctive data trade limits while evacuating HF-QRS. Most all of course, a rehash run something near 150 and $250 \mathrm{~Hz}$ has been exploited.

\section{Cancellation of higher frequency interference with ECG Signal}

\subsection{By using adaptive filter - conventional approaches on ANC}

Standard strategies of applying flexible channel to the decreasing or cancelation of high-repeat confusion used the structure. Two information sources are required in this structure: the fundamental data and reference input. Show the fundamental commitment as

$\mathrm{x}(\mathrm{n})=\mathrm{s}(\mathrm{n})+\mathrm{v}(\mathrm{n})$

Where $s(n)$ is the signal and $v(n)$ is the commotion. For our circumstance, $s(n)$ is the ideal ECG signal and $v$ (n) is the high repeat confusion. As an ANC, the adaptable channel has the errand to assess the commotion; that is, $y(n)=v^{\wedge}(n)$ for this circumstance,

$\mathrm{e}(\mathrm{n})=\mathrm{x}(\mathrm{n})-\mathrm{y}(\mathrm{n})=\mathrm{s}(\mathrm{n})+\mathrm{v}(\mathrm{n})-\mathrm{v}^{\wedge}(\mathrm{n}) \approx \mathrm{s}(\mathrm{n})$ 
Regardless, this is just the case at whatever point the reference data is connected with the change bit of the basic data; i.e., v(n). To show a related reference sign, lead devoted for taking the reference sign is set some place close to the position used to take the ECG signal. This may be an issue in light of the route that to have a reference hail all around related with the racket bit of the essential information is everything thought about not a direct undertaking. The motivation driving why this may happen is no under two-fallen. In any case, the section got by the presented lead may interface in some degree to the sign other than the aggravation. Second, the way utilized for passing the reference sign may harm the unsettling influence part got by the sensor. This as a general rule ruins the execution of the ANC. Fig.10, 11. Traces the standard technique of the ANC. The impact of the lead used to get the reference sign is tended to by a structure called L ( $\mathrm{z}$ ). For this situation the data to the versatile channel $\mathrm{r}(\mathrm{n})$ may not be all around related with $\mathrm{v}(\mathrm{n})$ and, in this manner, a phenomenal wiping out like (2) can't be conceivable.

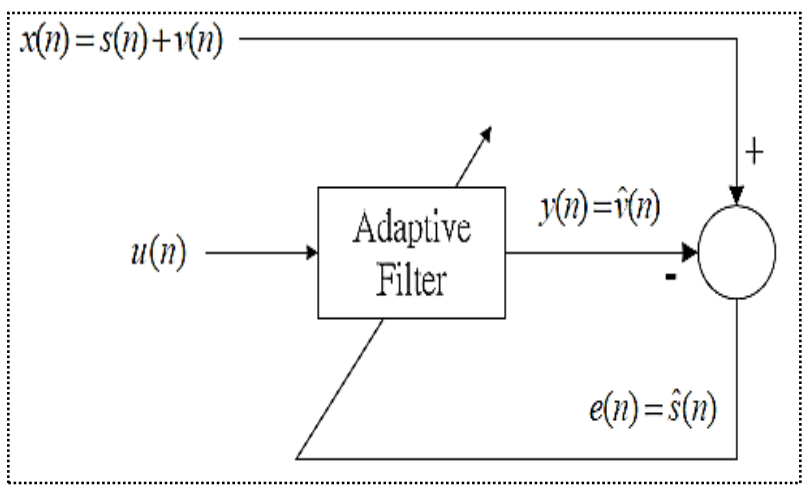

Fig. 10: Basic Model of ANC.

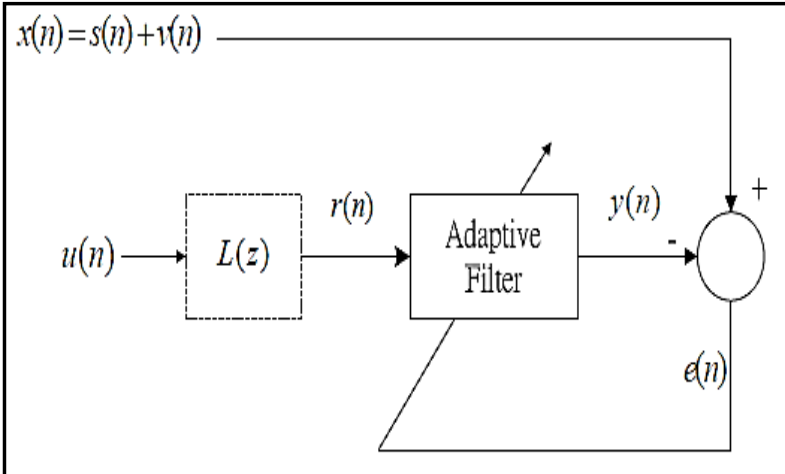

Fig. 11: ANC with Distorted External Reference.

\subsection{By using MATLAB}

To begin with, we will layout straightforward Butterworth channel and plot repeat using request frequency Fig.12, 13. By then we will check our expansion to confirm it meets specific. After that; we will apply our channel to the data signal

$x(n)=5 \cos (0.05 * \mathrm{pi} * \mathrm{n})+5 \cos (0.7 * \mathrm{pi} * \mathrm{n})$

At long last we will pot our information and out signal $\% \%$ title- - Outline channel [n, wn] = buttord $(0.1,0.4,-1,-20)$

$\mathrm{n}=2$

$\mathrm{wn}=0.1441$

$>>B, A]=\operatorname{butter}(n, w n)$

$B=0.0385 \quad 0.0770 \quad 0.0385$

$A=1.0000-1.3736 \quad 0.5275$

$\gg \operatorname{freq}(B, A)$

$>n=0: 0.1: 100$;

$>>x=5 * \cos (0.05 * p i * n)+0.1 * \operatorname{randn}(1,1001)$;

$>>p \operatorname{lot}(x)$

(Fig. (9))

$\gg y=\operatorname{filter}(B, A, x)$; 
$>\operatorname{plot}(y) \quad($ Fig. $(10))$

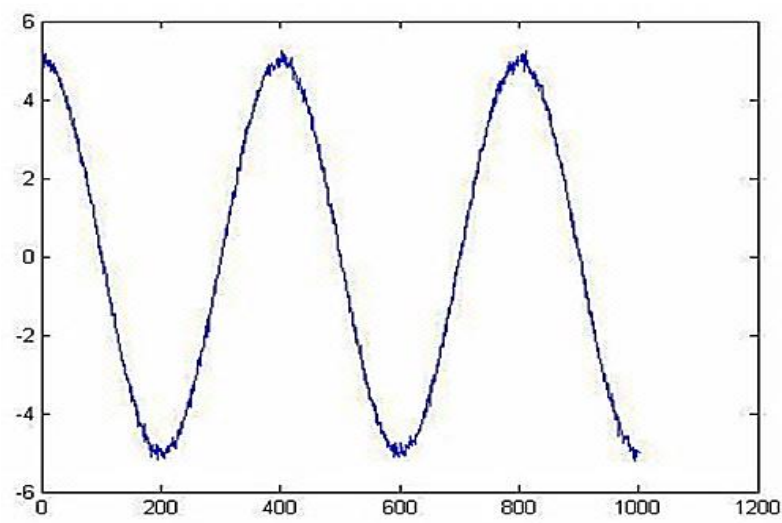

Fig. 12: Cosine Wave with Noise.

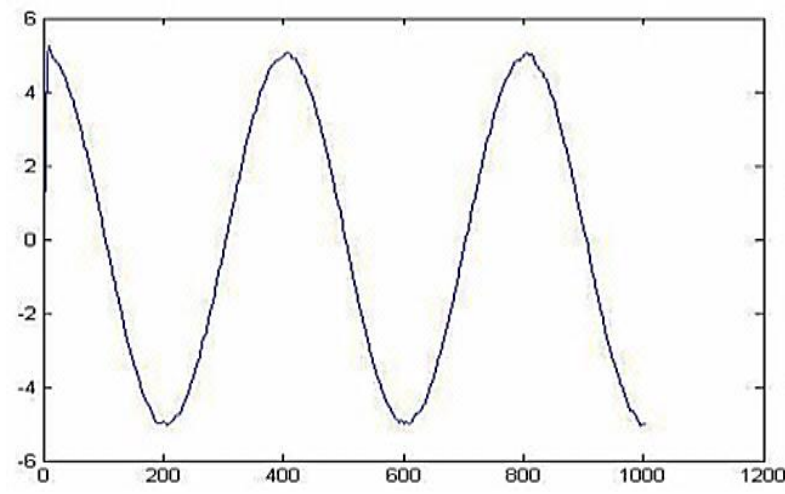

Fig. 13: Cosine Wave without Noise.

To mound the $118 \mathrm{e} 00 \mathrm{~m}$.mat that has two signals Fig. $(14,15)$ in the record (val $2 \times 3600$ ). We have to stack the second signal on MATLAB and open signal two that has 3600 core interests. We need to plot signal. Next, we have to use Fourier change to the signal by using charge FFT to have the ability to choose heart rate. Stack ECG signal; ECG signal= val (2, :); FFT= FFT (ECG signal); plot (ECG signal); plot (abs (FFT));

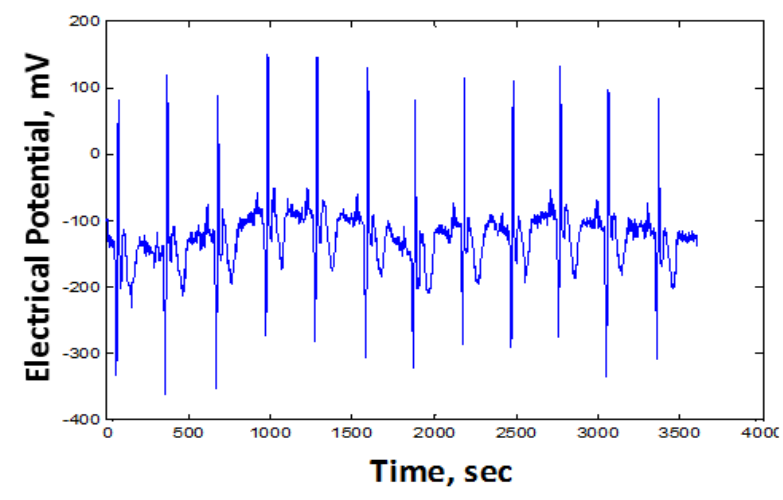

Fig. 14: Original ECG Signal.

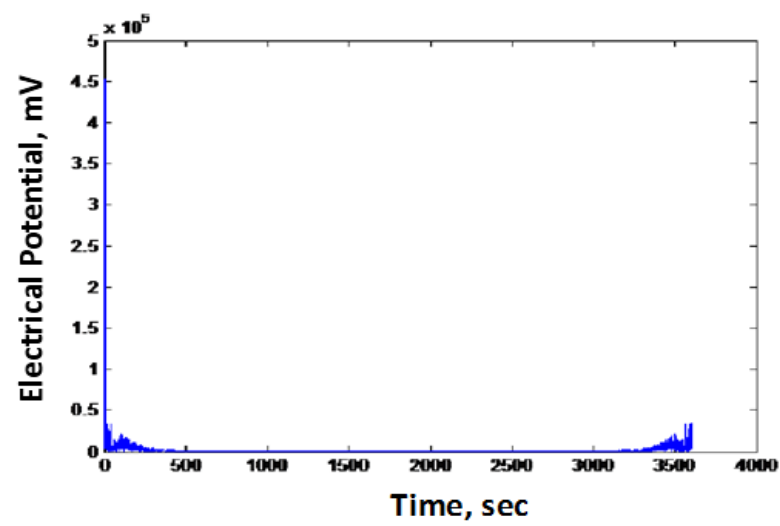

Fig. 15: FFT Signal. 
R-R period (it's the partition between two zeniths) and found out by choose the division between the most elevated purpose of fifth and best of sixth period by then subtract the detachment between after that we will diverse the result with 1/360 to find in second as show 1$1593-1290=303$

We different 303 to $1 / 360$ to get it in the second: The period $\mathrm{R}-\mathrm{R}$ in seconds is $303 * 1 / 360=0.844$ second

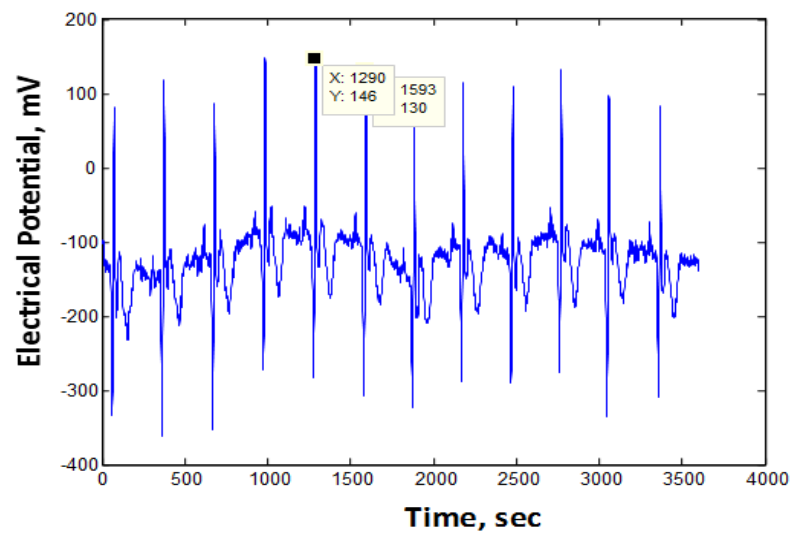

Fig. 16: R-R Period.

Exploiting the essential properties in Matlab to pick one ECG motion from the entire ECG motion in Fig.16. And take one epoch from it, the QRS appear in Fig.17. Ascertained as following;

$1-397-364=33$

$2-33 * 1 / 360=0.091$ seconds

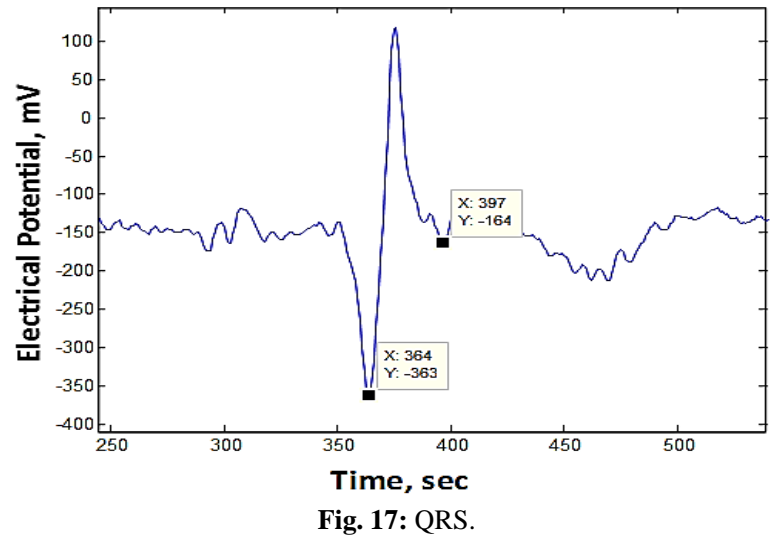

\subsection{Determine the heart rate using fourier transform (fft)}

Modelling just a single part from the Fourier variation in Fig.18. To decide the heart rate in the in the wake of utilizing FFT If: $180 \mathrm{HZ}$ 1800 examples X HZ 13 tests

In this way, $X=180 * 13 / 1800=1.3$ beat/second Thus, to get it in the minutes, it will be: $1.3 * 60=78$ beat for each moment.

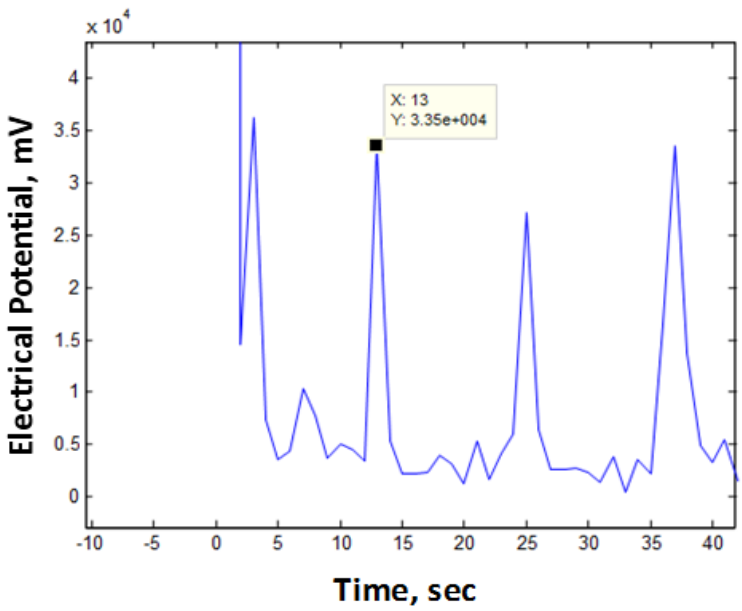

Fig. 1: The ECG Aft. 


\section{Conclusion}

A dispute occurred in the ANC with outside reference data is watched out for any disseminate of the outer information to the bustle part. Thusly, for the condition when outer reference sign isn't accessible, just ANC without outside reference data can be utilized. Since an impeccable ECG sign can be overseen as a quasiperiodic sign. By moving the uproarious ECG signal, its disarray segment can be made uncorrelated while leaving its sign area still remained related. By virtue of this property, an ANC without outer reference data is conceivable. Test results verified the sensibility of the new approach. We prescribed to build up our thought by reducing or scratching off the change in ECG structure by utilizing two system as an issue of first centrality we need to add to the versatile channel self-ruling from some other individual through refresh and change this sort of channel without require the reference hail also the adaptable channel require the unpredictable or rehash hail thusly we should see the ECG development as intermittent sign future work should manages these two matter of adaptable channel by evacuate the reference hail and succeeds any sort of sign broken or semi sporadic, furthermore, We actuate our companion who need to add to the strategy for getting or wiping out the bang by utilizing the thing estimation which can be turned out to be through the photograph and sign dealing with utilizing Matlab library work.

\section{Acknowledgment}

The authors wish to thank the Department of Mechanical, and Electrical \& Electronic Engineering, at Engineering College, University of Thi-Qar for its support.

\section{References}

[1] M. Marouf and L. Saranovac, "Adaptive EMG noise reduction in ECG signals using noise level approximation," in 2017 International Conference on Robotics and Machine Vision, 2017, vol. 10613, p. 106130E: International Society for Optics and Photonics. https://doi.org/10.1117/12.2299841.

[2] R. Adams, S. Demirtas, and J. G. Bernstein, "Time-domain interference removal for heart rate measurements," ed: Google Patents, 2018.

[3] R. Adams, S. Demirtas, and J. G. Bernstein, "Tracking mechanism for heart rate measurements," ed: Google Patents, 2018.

[4] S. Cuomo, R. Farina, and F. Piccialli, "An inverse Bayesian scheme for the denoising of ECG signals," Journal of Network and Computer Applications, 2018. https://doi.org/10.1016/j.jnca.2018.04.016.

[5] W. Wu, S. Pirbhulal, A. K. Sangaiah, S. C. Mukhopadhyay, and G. Li, "Optimization of signal quality over comfortability of textile electrodes for ECG monitoring in fog computing based medical applications," Future Generation Computer Systems, 2018. https://doi.org/10.1016/j.future.2018.04.024.

[6] J. A. Urigüen and B. Garcia-Zapirain, "EEG artifact removal-state-of-the-art and guidelines," Journal of neural engineering, vol. 12, no. 3, p. 031001, 2015. https://doi.org/10.1088/1741-2560/12/3/031001.

[7] D. Delisle-Rodriguez et al., "Adaptive Spatial Filter Based on Similarity Indices to Preserve the Neural Information on EEG Signals during OnLine Processing," Sensors, vol. 17, no. 12, p. 2725, 2017. https://doi.org/10.3390/s17122725.

[8] S. Patidar, R. B. Pachori, and U. R. Acharya, "Automated diagnosis of coronary artery disease using tunable-Q wavelet transform applied on heart rate signals," Knowledge-Based Systems, vol. 82,pp. 1-10, 2015. https://doi.org/10.1016/j.knosys.2015.02.011.

[9] E. J. Vigmond and B. D. Stuyvers, "Modeling our understanding of the His-Purkinje system," Progress in biophysics and molecular biology, vol. 120, no. 1-3, pp. 179-188, 2016. https://doi.org/10.1016/j.pbiomolbio.2015.12.013.

[10] A. S. Balcıoğlu and H. Müderrisoğlu, "Diabetes and cardiac autonomic neuropathy: clinical manifestations, cardiovascular consequences, diagnosis and treatment," World journal of diabetes, vol. 6, no. 1, p. 80, 2015. https://doi.org/10.4239/wjd.v6.i1.80.

[11] N. Schaerli et al., "P6373Incremental diagnostic value of high-frequency QRS analysis for the detection of exercise induced myocardial ischemia," European Heart Journal, vol. 38, no. suppl_1, 2017. https://doi.org/10.1093/eurheartj/ehx493.P6373.

[12] N. SruthiSudha and D. R. Reddy, "Detection and Removal of artefacts from EEG signal using sign based LMS Adaptive Filters," International Journal of Scientific \& Engineering Research, vol. 8, no. 2, pp. 950-954, 2017. https://doi.org/10.14299/ijser.2017.02.005.

[13] T. Schauer, "Sensing motion and muscle activity for feedback control of functional electrical stimulation: Ten years of experience in Berlin," Annual Reviews in Control, 2017. https://doi.org/10.1016/j.arcontrol.2017.09.014.

[14] R. Pilkar et al., "Application of empirical mode decomposition combined with notch filtering for interpretation of surface electromyograms during functional electrical stimulation," IEEE transactions on Neural Systems and Rehabilitation Engineering, vol. 25, no. 8, pp. 1268-1277, 2017. https://doi.org/10.1109/TNSRE.2016.2624763.

[15] P. Regalia, Adaptive IIR filtering in signal processing and control. Routledge, 2018. https://doi.org/10.1201/9781315136653.

[16] A. A. Khalaf, A. M. Said, M. Ibrahim, and H. Hamed, "Impact of Partial Update on Denoising Algorithms of ECG Signals," Journal of Telecommunication, Electronic and Computer Engineering (JTEC), vol. 10, no. 1-8, pp. 129-134, 2018.

[17] J. Xu, R. F. Yazicioglu, C. Van Hoof, and K. Makinwa, Low Power Active Electrode ICs for Wearable EEG Acquisition. Springer, 2018. https://doi.org/10.1007/978-3-319-74863-4.

[18] X. Liu et al., "A fully integrated wireless compressed sensing neural signal acquisition system for chronic recording and brain machine interface," IEEE Transactions on biomedical circuits and systems, vol. 10, no. 4, pp. 874-883, 2016. https://doi.org/10.1109/TBCAS.2016.2574362.

[19] A. Chan, China's workers under assault: Exploitation and abuse in a globalizing economy. Routledge, 2016. https://doi.org/10.4324/9781315502137.

[20] H. L. H. Chan, "Evaluating the Conditions for China's 4th Industrial Revolution Plan: A Neo-Schumpeterian Analysis," Singapore Management University (Singapore), 2016.

[21] Y. A. Altay and A. S. Kremlev, "Comparative analysis of ECG signal processing methods in the time-frequency domain," in Young Researchers in Electrical and Electronic Engineering (EIConRus), 2018 IEEE Conference of Russian, 2018, pp. 1058-1062: IEEE. https://doi.org/10.1109/EIConRus.2018.8317272.

[22] N. J. Stapelberg, D. L. Neumann, D. H. Shum, H. McConnell, and I. Hamilton-Craig, "A preprocessing tool for removing artifact from cardiac RR interval recordings using three-dimensional spatial distribution mapping," Psychophysiology, vol. 53, no. 4, pp. 482-492, 2016. https://doi.org/10.1111/psyp.12598.

[23] K. K. Muhsin. (2008). Frequency Analysis of Articulated Robot. Basra Journal for Engineering Science ,University of Basrah, 8(1), 90-108.

[24] K, Mushin, " Modeling Of Structural Human Dynamic Response ", Thi-Qar University Journal for Engineering Sciences, Babylon University, vol. 7, no. 2, pp. 1-17, 2016.

[25] K. K. Mohsen, "Spectrum Analysis of The Gate of Dike Structure Under Nonstationary Random Loading "Thi-Qar University Journal for Engineering Sciences, Thi-Qar University,vol.1, no. 1, pp. 48-62., 2010 